\title{
EL SACRIFICIO HUMANO POR EXTRACCIÓN DE CORAZÓN. UNA EVALUACIÓN OSTEOTAFONÓMICA DE VIOLENCIA RITUAL ENTRE LOS MAYAS DEL CLÁSICO*
}

\author{
Vera Tiesler y Andrea Cucina \\ Facultad de Ciencias Antropológicas \\ Universidad Autónoma de Yucatán
}

\section{Introducción}

El sacrificio humano era considerado la expresión suprema del ejercicio religioso entre los mayas. En particular, la remoción del corazón pulsante, la inmolación de la vida humana y el ofrecimiento del órgano vital —esencia y alimento de los dioses- permitía la recompensa de lo sagrado y la comunicación última con ello (Nájera, 1987: 9, 40-50). Al igual que otras prácticas sacrificiales, la extracción del corazón era regulada por un conjunto de normas y reglas específicas (Nájera, 1987: 144-165; López Austin, 1989: 432-442; Carrasco, 1999: 81-87). Los procedimientos sacrificiales incluían la preparación de los oficiantes y de la víctima, la apertura violenta del tronco y la ablación y consagración del corazón. En seguida, el cuerpo, ahora parte de lo sagrado, podía ser sometido a diferentes tratamientos como la mutilación, el canibalismo ritual o el enterramiento en lugares específicos (Schele, 1984: 7-8; Nájera, 1987: 205-216; Taube, 1999: 228-230; véase también Matos Moctezuma, 1986: 105-118; López Austin, 1989: 432-442).

Prácticas de extracción cardiaca están muy bien documentadas durante las épocas Posclásica y Colonial en el área maya; menos conocidos son los procedi-

\footnotetext{
* Queremos agradecer a los siguientes proyectos y colegas por el acceso a materiales, las detenidas discusiones, los consejos e información: Proyecto Restos Humanos del Templo XIII, Palenque, Chiapas (a cargo de Arturo Romano Pacheco), Arnoldo Cruz González (Proyecto Arqueológico Palenque), Proyecto Arqueológico Becán (a cargo de Luz Evelia Campaña), Proyecto Arqueológico Calakmul (a cargo de Ramón Carrasco Vargas), Sergio Raúl Arroyo (director del INAH), Francisco Ortiz Pedraza (Dirección de Antropología Física, INAH), Margaret Streeter (Department of Anthropology, University of Missouri at Columbia), Iván Olivia y Patricia Quintana (CINVESTAV, Politécnico Nacional, Unidad Mérida). Gracias a Felipe Bate Petersen (Escuela Nacional de Antropología e Historia, INAH) por asistirnos en la identificación de los implementos cortantes. Apreciamos mucho también la ayuda y asistencia de Wilberth Pantoja (SEMEFO), Margarita Valencia (SEMEFO) y Rafael Blázquez (Centro Médico Nacional Ignacio Téllez García, IMSS); gracias por compartir su amplia experiencia y conocimiento en materia de patología forense y cardiocirugía con nosotros.
} 
mientos empleados en tiempos anteriores (Taube, 1999: 228-2330; Miller, 2003: 383-400), una situación que es extensiva también a otras áreas mesoamericanas. Si bien gracias a la iconografía se reconoce que la remoción ritual del corazón constituía una práctica ya conocida durante el Clásico (Robicsek y Hales, 1984: 49), se considera que la decapitación era la forma más común de muerte ritual durante el primer milenio d. C. pues predomina claramente en el acervo iconográfico sacrificatorio procedente de aquel tiempo (Miller y Taube, 1993).

Hasta el momento, los estudios sobre las prácticas rituales se han basado mayormente en la documentación etnohistórica y en el registro gráfico (Schele, 1984: 7; Nájera, 1987: 10; Schele y Miller, 1986: 15-16). Entretanto, la identificación arqueológica de las víctimas sacrificiales aún se limita a indicios indirectos que derivan de la información contextual: la presencia de entierros simultáneos múltiples e irregulares, caracterizados por un perfil de sexo y edad no demográfico o por la ausencia de objetos funerarios asociados. Aun menos peso se ha concedido a la evidencia de violencia que puede encontrarse en los esqueletos, si bien posee un gran potencial para la reconstrucción de procesos rituales que involucraban al cuerpo humano (Massey y Steele, 1997: 62-77; Tiesler, 2002a: 137-139; Tiesler, et.al.: 75-77; Buikstra, et al., 2004: 202-203; Tiesler y Cucina, 2003: 337).

En el caso específico de la práctica de remoción cardiaca, el mal estado de preservación de los restos arqueológicos humanos, producto del ambiente húmedo y tropical que caracteriza gran parte del área maya, no facilita el registro directo de los huesos y limita o impide un acercamiento osteotafonómico, entendido en este caso como la evaluación integral del esqueleto en su contexto deposicional (Tiesler, 2003a). De hecho, tampoco en otras áreas de Mesoamérica abundan las marcas esqueléticas que pudieran ser producto del sacrificio por extracción cardiaca. Los huesos presumiblemente afectados, es decir, las costillas y el esternón (cuerpo y manubrio), raramente presentan marcas tangibles de apertura torácica. Aquellos que sí las presentan, como son los esternones cortados de Champotón, Campeche, Loma Alta, Michoacán, y Tlatelolco, en la Ciudad de México (Pijoan, 1997: 242-280; Pereira, 1999: 233-243; Gómez, et al., 2003: 146147), están acompañados de marcas de manipulación claramente póstumas, por lo cual no puede asegurarse que hayan sido producto del sacrificio o acaso de algún tratamiento posterior a la muerte.

Los procedimientos técnicos de la intervención ritual seguramente variaban de región en región. En 1984, Robicsek y Hales afirmaron, tras una discusión de las ventajas y desventajas de cuatro procedimientos, que el acercamiento al corazón se debe haber logrado mediante una toracotomía transversal bilateral, implicando que el corte cruzaba el tórax de lado a lado, abriendo el esternón al nivel de la quinta o sexta costilla. Los autores abogan por este acercamiento fundamentándose en los beneficios que conlleva en la práctica quirúrgica actual, además de citar e interpretar una serie de escenas sacrificiales representadas en la iconografía prehispánica así como la documentación histórica de la región. Concluyen 
con ello que el acercamiento propuesto debió haber sido la técnica de apertura más factible, rápida y directa (figura 1) al ocasionar un colapso inmediato de los pulmones de la víctima y con ello el desmayo, posibilitando así un acceso al corazón después de tres a cuatro minutos. A diferencia de Robicsek y Hales, otros investigadores admiten la posibilidad de diferentes acercamientos (véase, por ejemplo, González Torres, 1985: 113-116) y alegan que en el área maya se privilegiaba el acceso por debajo de la caja torácica. Igualmente, Pereira (1999: 192-194) propone que para el área de Loma Alta, Michoacán, el acceso habría sido subtorácico.

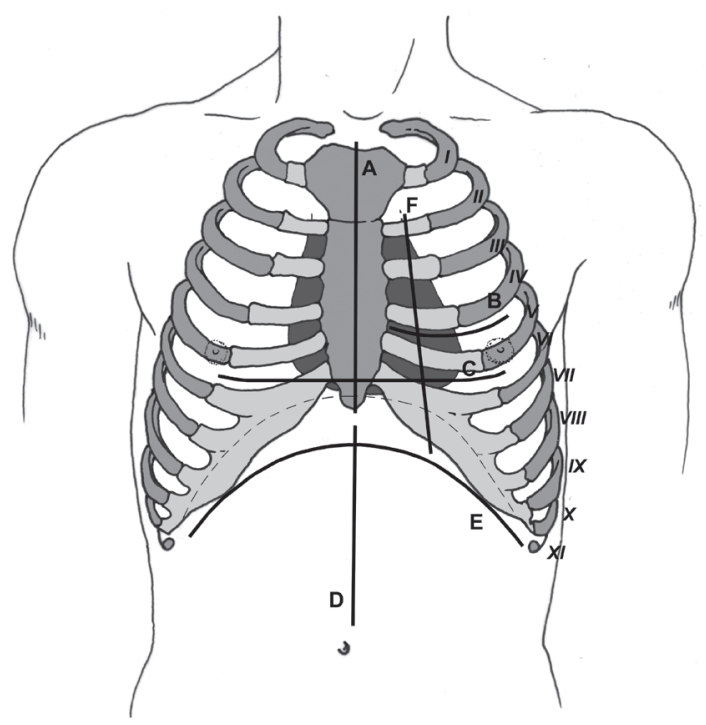

Figura 1. RePRESENTACIÓN GRÁfiCA DE LOS DifERENTES ACERCAMIENTOS Al CORAZÓN.

Caja torácica en posición sobre extendida con el diafragma representado mediante una línea punteada. A) estereotomía vertical axial;

B) toracotomía transversal izquierda; C) toracotomía bilateral transversal;

D) acceso transabdominal vertical; E) acercamiento subtorácico transdiafragmático;

F) acceso paraesternal (dibujo de Vera Tiesler).

En el presente trabajo deseamos contribuir al debate sobre la extracción ritual del corazón entre los mayas con una serie de criterios operacionales fundamentados directamente en la evidencia esquelética. Proponemos para ello un modelo que integra un conjunto de pasos para la extracción del órgano, los cuales permiten reconstruir el proceso ritual y correlacionarlo con las potenciales marcas encontradas. En concreto, este modelo trata de explicar la conducta que originó las marcas de violencia perimortem encontradas en restos esqueléticos primarios 
de Palenque, Calakmul y Becán, en México, así como en un potencial caso hallado en los restos de los acompañantes de Pakal en el Templo de las Inscripciones en Palenque. Aparte de la interpretación de dichos casos, deseamos ofrecer una explicación plausible sobre la reducida presencia en la región de marcas esqueléticas correspondientes al sacrificio cardiaco.

\section{Marcas de violencia perimortem y manipulación póstuma del cuerpo en los sitios de Palenque, Calakmul y Becán}

Al principio, nuestras reflexiones sobre el patrón de la excisión cardiaca ritual fueron impulsadas por la evidencia encontrada en cuatro esqueletos humanos, todos procedentes de contextos primarios. Fueron hallados en las estructuras centrales de los sitios clásicos de Palenque, Calakmul y Becán en México. Tres de ellos se asocian con cámaras sepulcrales.

Las marcas culturales fueron investigadas directamente por los autores en cada uno de los contextos y en las evaluaciones osteotafonómicas llevadas a cabo utilizando la información fotográfica y/o los dibujos tafonómicos. Anteriormente, las muestras habían sido cuidadosamente restauradas por profesionales.

El sexo y la edad de los individuos fueron estimados con base en los indicadores macroscópicos analizados en pelvis, fémures, mandíbulas y cráneos (Ubelaker, 1989; Meindl, et al., 1985; Iscan y Loth, 1986; Brooks y Suchey, 1990; Buikstra y Ubelaker, 1994), mientras la evaluación tafonómica siguió lo establecido por Duday (et al., 1990; Duday, 1997). Las huellas de impacto fueron analizadas bajo luz tangencial utilizando un microscopio estereoscópico de 10 a 40 aumentos.

Nuestro primer caso procede de Calakmul, Campeche. Corresponde a una mujer que murió en su tercera década de vida y fue enterrada en la antecámara de la tumba dinástica sub-4a en la Estructura II (Carrasco, et al., 1999: 47-59; Tiesler, 2003a). Con base en los elementos iconográficos y en su edad al morir, se deduce que la mujer, junto con un niño de entre 10 a 12 años de edad, acompañaba a quien fue identificado como el gobernante Yuknoom Yich'aak K'ak'. Las inscripciones nos narran que el jerarca ascendió al trono en el $686 \mathrm{~d}$. C., y se piensa que murió al finalizar el siglo (Carrasco, et al., 1999: 47-59; Martin y Grube, 2000: 110-111; Carrasco, 2000: 12-21; García, Moreno y Granados, 2000: 28-33). La acompañante, en deposición primaria, yacía en posición extendida y estaba rodeada de una gran cantidad de adornos personales y objetos funerarios, además del cuerpo del niño arriba mencionado. El análisis tafonómico llevado a cabo durante la excavación permitió establecer que los dos individuos fueron depositados simultáneamente y durante el mismo evento en el cual fue emplazado el personaje dentro de la cámara principal (Tiesler, 2003a; Ramón Carrasco, comunicación personal, 2005).

Las marcas encontradas en la mujer se pueden observar en la figura 2a. Se trata de uno o probablemente dos cortes en la porción inferior izquierda de 
la duodécima vértebra torácica. El corte presenta márgenes bien delimitados y regulares y penetra el hueso entre dos y cuatro milímetros. Aparentemente, el arma presentaba un filo recto que, más que por deslizamiento, impactó directamente sobre los segmentos (Bate, comunicación personal, 2003). Ninguna otra vértebra presentaba marcas, pero aclaramos que el cuerpo de las primeras vértebras lumbares no se preservaron, por lo cual no es posible establecer el patrón de los cortes debajo de la doceava torácica.

Los casos de Palenque, Chiapas, conciernen respectivamente a una joven mujer que acompañaba los restos mortales de la Reina Roja en el Templo XIII-sub (López Jiménez y González Cruz, 1995: 124-125; González Cruz, 1998: 61, 2001; Tiesler, et al., 2002: 76; Cucina, et al., 2004: 82) y a uno de los cuerpos depositados en el contexto múltiple que sellaba la entrada de la cámara del rey Janaab' Pakal en el Templo de las Inscripciones.

La mujer hallada en el Templo XIII-sub fue descubierta en la cámara sepulcral al lado del sarcófago con los restos de la Reina Roja, una alta dignataria cuya identidad sigue sin establecerse (Tiesler, et al., 2004: 74).* Su cuerpo estaba depositado en posición ventral con los brazos cruzados atrás. Alteraciones tafonómicas posdeposicionales no permiten hacer inferencias precisas sobre la correspondencia anatómica del cuerpo; sin embargo, la porción superior del tronco se halla desfasada lateralmente en relación con la parte inferior, lo cual da la impresión de que el cuerpo fue partido en dos a nivel del abdomen (Tiesler, et al., 2002: 77). La investigación macroscópica evidenció numerosas marcas de impacto con arma cortante en el área torácico-lumbar. Dos costillas, que por sus dimensiónes y características deben ubicarse entre la número 7 y la 10, ostentan profundos surcos que afectan el hueso a unos centímetros de la articulación costo-vertebral (Tiesler y Cucina, 2003: 341-242). Sus características indican que fueron provocados por una serie de impactos punzo-cortantes. Tres vértebras torácicas bajas (T10, T11 y T12), así como la tercera vértebra lumbar, presentan numerosos vestigios de violencia perimortem. La onceava vértebra torácica resulta ser la más lesionada durante este acto. Las porciones derecha y superior del cuerpo fueron seccionadas mediante un impacto cortante. En general, el patrón de las huellas y los golpes inferibles a partir de los restos es propio de un proceso violento de manipulación del cuerpo llevado a cabo desde adelante y atrás, quizá con la intención de separar el cuerpo en dos. Respecto al presente estudio, las marcas de interés se encuentran en el margen inferior derecho del onceavo cuerpo vertebral (figura 2b). Esta huella, señalada por la flecha en la figura, presenta un patrón muy bien delineado; con ello podemos excluir que haya sido el resultado de procesos tafonómicos póstumos.

El segundo caso de Palenque corresponde a uno de los cinco individuos hallados en la caja que sellaba la cámara funeraria de Janaab’ Pakal (Ruz, 1973: 54-56;

\footnotetext{
* Nota del editor: después de una acuciosa investigación dirigida por la misma Vera Tiesler, se concluyó que la persona enterrada en la Tumba Roja fue la señora Sak B'u Ajaw, esposa de Kinich Janab' Pakal.
} 
véase también Cucina, et al., 2004: 81). Los restos se encontraron completamente empotrados en concreciones calcáreas que impidieron un análisis macroscópico sistemático. Aun así se reconoció una décima vértebra torácica con una huella de impacto en su margen antero-superior (figura 2c). El margen inferior presenta una rotura reciente mientras más arriba se reconoce una lesión, con márgenes bien definidos, que aún estaba cubierta por concreciones al igual que el resto de la superficie. Este último aspecto podría indicar que la huella fue producida antes de la deposición calcárea y al mismo tiempo excluye su origen moderno, como podría haber sido la intervención efectuada durante la recuperación de los restos en el año 1952. Desafortunadamente, no fue posible estudiar todos los segmentos óseos de los acompañantes de Pakal. Esta limitación, junto con la mala preservación del material, nos induce a considerar este caso sólo como un posible indicador del ritual de sacrificio aquí estudiado.

El último espécimen que nos ocupa fue descubierto debajo de la escalera en una subestructura clausurada dentro del Complejo X de Becán, Campeche (Tiesler, 2002b; véase también Tiesler y Campaña, 2006: 32-46). El cuerpo pertenecía a un adolescente de entre 15 y 18 años de edad, probablemente de sexo masculino (Buikstra y Ubelaker, 1994) y que yacía en posición flexionada. Los análisis tafonómicos indican que la osamenta fue visitada tiempo después de que la cámara había sido sellada para extraer algunos huesos. Pese a esta remoción (principalmente de los huesos largos y el cráneo), la mayoría de los segmentos torácicos, la pelvis y los pies mantenían todavía su conexión anatómica. Ahora bien, el lado izquierdo del cuerpo de la duodécima vértebra torácica presentaba tres profundas muescas verticales con bordes regulares (figura 2d). Tal como en los casos restantes, el corte parece haber sido causado más por impacto que por deslizamiento (Bate, comunicación personal, 2003). Aparentemente, el proceso de violencia perimortem no afectó más partes, pues no se detectaron huellas de violencia en otros segmentos observables (Tiesler y Campaña, 2006: 32-46).

Ahora bien, las evidencias apuntan a que ninguna de las marcas culturales arriba descritas fueron el producto de procesos tafonómicos ocurridos durante la fase de recuperación y restauración del material. Tampoco creemos que se hayan producido después de la perturbación del esqueleto, ya que no sería factible producir marcas de las presentes características en osamentas. En el caso del hueso seco, la presión y violencia necesarias para provocar estas huellas habría fragmentado la pieza en vez de dejar surcos y habría alterado notablemente su aspecto.

Las marcas encontradas en los cuatro especímenes analizados presentan un patrón común. En todos los casos, el área afectada corresponde a las últimas vértebras torácicas, las secciones se muestran bien marcadas y regulares y el tejido óseo luce comprimido, lo cual sugiere un patrón de impacto en hueso fresco con un instrumento filoso ancho. Además, la porción del cuerpo vertebral afectada resulta ser la izquierda en todos los casos.

A la luz de las semejanzas encontradas, nos enfocaremos en la tarea de reconstruir, desde una perspectiva metodológica, los procedimientos que podrían 


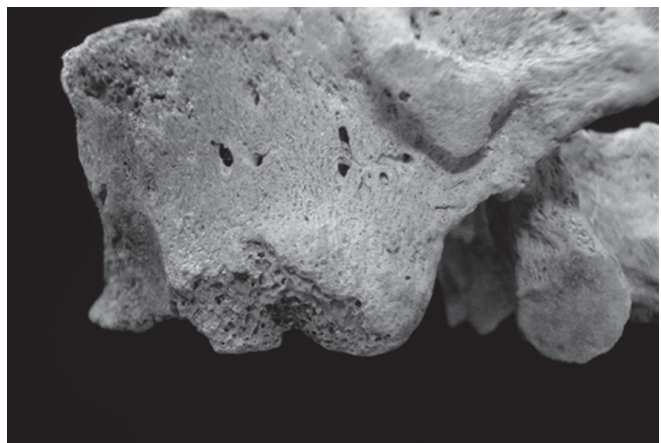

a)

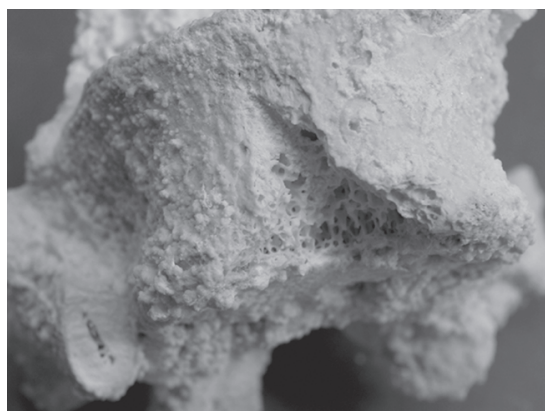

c)

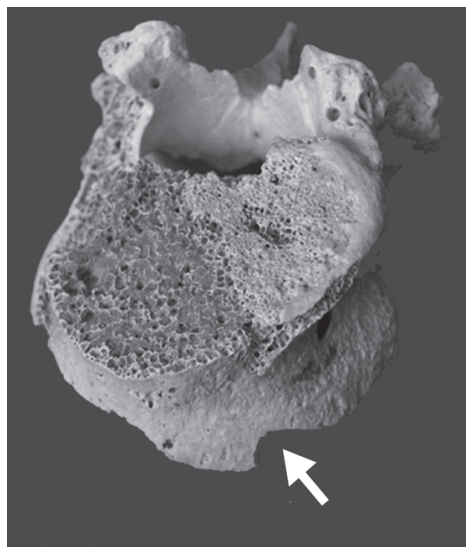

b)

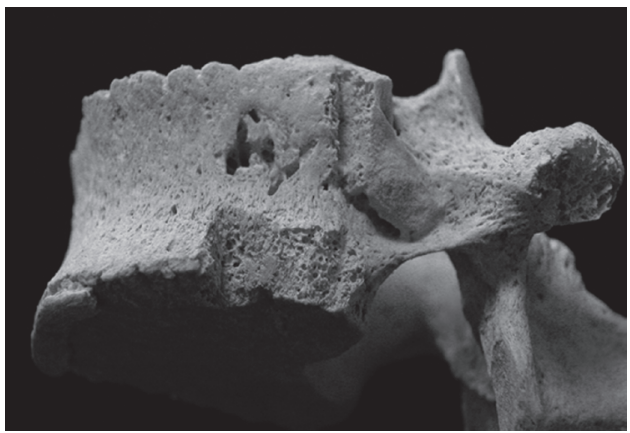

d)

Figura 2. MARCAS DE CORTE POR IMPACTO en la porción izquierda en los cuerpos de las últimas vértebras torácicas procedentes de: a) Calakmul;

b) Palenque (Templo XIII-sub: acompañante de la Reina Roja);

c) Palenque (Templo de las Inscripciones:

uno de los acompañantes de Janaab' Pakal);

d) Becán (fotos de Andrea Cucina). 
dar cuenta de las lesiones e interpretarlas conjuntamente con la evidencia osteoarqueológica, etnohistórica e iconográfica. Discutimos nuestros resultados subrayando sus implicaciones en la investigación de los patrones funerarios mayas y las conductas sacrificiales durante el Clásico, como parte de un culto personal dirigido a y por la antigua aristocracia.

\section{Procedimientos y pasos rituales}

A fin de poder diferenciar actos de violencia perimortem de aquellos tratamientos que ocurrieron en los conjuntos esqueléticos en una fase póstuma, la interpretación osteotafonómica y contextual busca reconocer patrones y regularidades. La violencia perimortem designa por definición un evento que acontece entorno a la muerte, y cuando afecta al esqueleto suele resultar en huellas por impacto sin sanar y se distinguen de los patrones que dejan las marcas claramente póstumas, tales como los cortes por desmembramiento, descarnamiento o exposición al fuego por largo tiempo.

Considerando que la tradición de la extracción del corazón, al igual que otras prácticas religiosas, sigue un patrón establecido y altamente pautado, es plausible suponer que las potenciales marcas dejadas por aquella en el esqueleto deben también presentar un patrón compartido. Para la presente reconstrucción de los procedimientos involucrados tomamos en cuenta todos los parámetros que se sabe jugaban un papel en el evento ritual: el instrumental utilizado y la rapidez en la ejecución del acto por un ejecutor ayudado por varios asistentes (Nájera, 1987: 143-150; para Mesomérica en general, véase también González Torres, 1985, 2003: 40; López Austin, 1998: 11). Landa (1982) menciona que el nacón se acercaba a la víctima con un cuchillo de pedernal, cortaba súbitamente el lado izquierdo debajo del pecho para insertar la mano y extraer el corazón pulsante. Tomas López-Méndez (citado en Tozzer, 1941) también subraya la rapidez y facilidad con la cual se llevaba a cabo el acto. El nacón se caracteriza en las descripciones como la única persona que ejecutaba la escisión, mientras unos asistentes mantenían el cuerpo extendido sobre el altar (Scholes y Adams, 1938; véase Anda, et al., 2004: 379). Acto seguido, aquel entregaba el corazón al sacerdote Ah k'in para su consagración y presentación a los dioses.

Para nuestros propósitos de reconstrucción conductual recurrimos al esquema conceptual de la expresión ritual del sacrificio formulado por López Austin (1998: 15), que permite separar los pasos rituales documentados en una serie de componentes básicos (tabla 1). Las categorías representadas en la tabla se proponen dilucidar la compleja serie de pasos que preparan, constituyen y siguen al acto culminante del sacrificio. Dicha clasificación pretende facilitar la correlación entre el comportamiento ritual y las marcas en el esqueleto que la intervención pudo haber dejado. 
Por lo pronto, las semejanzas encontradas en los cuatro casos aquí analizados (donde se afecta la porción ventral izquierda de las últimas vértebras torácicas) inducen a considerar que son el producto de un procedimiento común. Debido a que los cuerpos de las víctimas estaban intactos, el instrumento debió penetrar el tórax para poder afectar las vértebras. La iconografía y los registros coloniales suministran información que apoya la interpretación de las marcas, pues describen dos acciones rituales capaces de explicar la presencia de las huellas: la extracción del corazón y la remoción de las vísceras (Schele, 1984: 7; Schele y Miller, 1986: 217; Nájera, 1987: 147-150; Stuart, 2003: 25-26).

Crucial para la verificación de ambas posibilidades es la topografía de las lesiones en el cuerpo y sus relaciones anatómicas con los tejidos que las circundan.

Tabla 1. Pasos Rituales y EVIDENCia osteotafonómica esPerada

\begin{tabular}{|c|c|c|c|}
\hline Pasos rituales & Procedimientos & $\begin{array}{l}\text { Probable utensilio } \\
\text { empleado }\end{array}$ & $\begin{array}{l}\text { Posible evidencia } \\
\text { esquelética }\end{array}$ \\
\hline $\begin{array}{l}\text { Dispersión } \\
\text { de sangre } \\
\text { anterior a la } \\
\text { muerte }\end{array}$ & $\begin{array}{l}\text { Boca, nariz, ore- } \\
\text { jas, dedos, pene }\end{array}$ & $\begin{array}{l}\text { Espina de mantaraya, } \\
\text { lasca }\end{array}$ & Ninguna \\
\hline $\begin{array}{l}\text { Colocación de } \\
\text { la víctima }\end{array}$ & $\begin{array}{l}\text { Altar, piedra } \\
\text { ritual }\end{array}$ & $\begin{array}{l}\text { Monolito, estructura } \\
\text { en madera }\end{array}$ & Ninguna \\
\hline \multirow{6}{*}{$\begin{array}{l}\text { Acceso } \\
\text { al corazón }\end{array}$} & $\begin{array}{l}\text { (A) Esternotomía } \\
\text { axial vertical }\end{array}$ & $\begin{array}{l}\text { (A) Piedra para serru- } \\
\text { char, lasca o cuchi- } \\
\text { llo bifacial, hacha, } \\
\text { martillo }\end{array}$ & $\begin{array}{l}\text { (A) Seccionamiento o } \\
\text { fractura del esternón }\end{array}$ \\
\hline & $\begin{array}{l}\text { (B) Toracoto- } \\
\text { mía transversal } \\
\text { izquierda }\end{array}$ & $\begin{array}{l}\text { (B) Lasca o cuchillo } \\
\text { bifacial }\end{array}$ & $\begin{array}{l}\text { (B) Marcas de corte } \\
\text { transversal del esternón, } \\
\text { posición medial izquierda }\end{array}$ \\
\hline & $\begin{array}{l}\text { (C) Esternotora- } \\
\text { cotomía bilateral } \\
\text { transversal }\end{array}$ & $\begin{array}{l}\text { (C) Piedra para serru- } \\
\text { char }\end{array}$ & (C) Bisección del esternón \\
\hline & $\begin{array}{l}\text { (D) Acceso } \\
\text { transdiafragmá- } \\
\text { tico abdominal } \\
\text { vertical }\end{array}$ & $\begin{array}{l}\text { (D) Lasca o cuchillo } \\
\text { bifacial }\end{array}$ & $\begin{array}{l}\text { (D) Ninguna o marcas } \\
\text { tangenciales en la porción } \\
\text { inferior del esternón o de } \\
\text { las costillas }\end{array}$ \\
\hline & $\begin{array}{l}\text { (E) Acceso subto- } \\
\text { rácico transdia- } \\
\text { fragmático }\end{array}$ & $\begin{array}{l}\text { (E) Lasca o cuchillo } \\
\text { bifacial }\end{array}$ & $\begin{array}{l}\text { (E) Ninguna o marcas } \\
\text { tangenciales en la porción } \\
\text { inferior del esternón o de } \\
\text { las últimas costillas }\end{array}$ \\
\hline & $\begin{array}{l}\text { (F) Acceso } \\
\text { paraesternal } \\
\text { izquierdo }\end{array}$ & $\begin{array}{l}\text { (F) Cuchillo bifacial o } \\
\text { piedra para serruchar }\end{array}$ & (F) Ninguna \\
\hline
\end{tabular}




\begin{tabular}{|l|l|l|l|}
\hline $\begin{array}{l}\text { Exposición } \\
\text { del corazón }\end{array}$ & (A) Transtorácico & $\begin{array}{l}\text { (A) Utensilios de } \\
\text { impacto: dilatador de } \\
\text { costillas, hachas }\end{array}$ & $\begin{array}{l}\text { (A) Marcas de corte o } \\
\text { impacto en las costillas, } \\
\text { esternón y cuerpos verte- } \\
\text { brales (impacto directo) }\end{array}$ \\
\cline { 2 - 4 } & $\begin{array}{l}\text { (B) Transdiafrag- } \\
\text { mático }\end{array}$ & (B) Cuchillo bifacial & (B) Ninguna \\
\hline $\begin{array}{l}\text { Remoción } \\
\text { del corazón }\end{array}$ & $\begin{array}{l}\text { Corte de los } \\
\text { ligamentos }\end{array}$ & Cuchillo bifacial & $\begin{array}{l}\text { Ninguna o marcas tan- } \\
\text { genciales en las costillas, } \\
\text { esternón y vértebras }\end{array}$ \\
\hline $\begin{array}{l}\text { Ofrenda } \\
\text { del corazón }\end{array}$ & $\begin{array}{l}\text { Posicionamiento, } \\
\text { incineración }\end{array}$ & $\begin{array}{l}\text { Vasos, quemador de } \\
\text { incienso, fuego }\end{array}$ & $\begin{array}{l}\text { Ninguna o huellas de } \\
\text { residuos químicos }\end{array}$ \\
\hline $\begin{array}{l}\text { Ofrenda } \\
\text { de sangre } \\
\text { un vaso, de- } \\
\text { rramamiento, } \\
\text { incineración }\end{array}$ & $\begin{array}{l}\text { Vasos, quemador de } \\
\text { incienso, fuego }\end{array}$ & $\begin{array}{l}\text { Ninguna o huellas de } \\
\text { residuos químicos }\end{array}$ \\
\hline $\begin{array}{l}\text { Mutilación } \\
\text { sacrificial } \\
\text { del cuerpo }\end{array}$ & $\begin{array}{l}\text { Carnicería, des- } \\
\text { membramiento, } \\
\text { cremación }\end{array}$ & $\begin{array}{l}\text { Fuego y utensilios de } \\
\text { impacto: cuchillos y } \\
\text { hachas }\end{array}$ & $\begin{array}{l}\text { Exposición al calor, } \\
\text { marcas de golpe, de corte } \\
\text { o de impacto, fracturas } \\
\text { aisladas. Las huellas se } \\
\text { encuentran principalmen- } \\
\text { te en los apéndices }\end{array}$ \\
\hline $\begin{array}{l}\text { Tratamiento } \\
\text { póstumo } \\
\text { del cuerpo } \\
\text { térmico, des- } \\
\text { carnamiento, } \\
\text { desollamiento, } \\
\text { reutilización }\end{array}$ & $\begin{array}{l}\text { Fuego y utensilios } \\
\text { de precisión: lascas, } \\
\text { cuchillos bifaciales, } \\
\text { pulidores, etc. }\end{array}$ & $\begin{array}{l}\text { Exposición al calor, mar- } \\
\text { cas de golpe, percusión, } \\
\text { fracturas extensas, cortes } \\
\text { de precisión, marcas en } \\
\text { todo el cuerpo }\end{array}$ \\
\hline
\end{tabular}

Las marcas están ubicadas en el lado izquierdo, en la porción posterior interna de la cavidad torácica y por encima de la inserción fibrosa del diafragma (que separa el tórax del abdomen). La topografía orgánica relega los tajos al mediastino inferior de la cavidad torácica más que a la abdominal, razón por la cual descartamos la hipótesis de una remoción abrupta de los órganos abdominales. Aun menor es la probabilidad de que el proceso de evisceración descrito para el Posclásico (Ruz, 1991) haya dejado las lesiones profundas presenciadas en este estudio. Era de esperarse que la remoción de las vísceras hubiera dejado su huella más bien en forma de sutiles deslizamientos en columna, pelvis y caja torácica (M. Valencia, semefo, comunicación personal, 2004).

Evaluemos entonces las implicaciones de conducta del segundo acto, $i$. e. la extracción de corazón. Aparentemente, las marcas encontradas no son el producto de la apertura directa del tórax, sino del corte violento de tejidos en la cavidad pectoral. La ausencia de huellas en el esternón sugiere que el acceso pudo haber sido intercostal, paraesternal o por debajo de la caja torácica (figura 1 
y tabla 1; procedimientos $\mathrm{B}, \mathrm{D}, \mathrm{E}, \mathrm{F})$. Consideramos que el acceso a través de las costillas no proporcionaría espacio suficiente para remover el corazón, además de requerir un tiempo relativamente prolongado y la asistencia directa de otras personas, como ya han subrayado Robicsek y Hales (1984: 82). Los mismos problemas se manifestarían en una intervención paraesternal vertical, además de que este acercamiento requiere también el corte del cartílago costoesternal. Ninguno de los dos parece proporcionar un método viable, fácil y sobre todo de acceso rápido al corazón. Igualmente improbable sería una aproximación subdiafragmática vertical, la cual, a pesar de no implicar huesos o cartílagos, encontraría en el camino los tejidos intestinales.

Por otra parte, la aproximación al corazón desde el margen inferior de las costillas (procedimiento E) parece resultar el modo más directo y rápido una vez que el cuerpo está en posición supina sobreextendida. No requiere de la sección de huesos o cartílagos, y en el caso de individuos femeninos, como los que se presentan en este estudio, tampoco interfiere con el seno. Una vez seccionado el diafragma en el acto de apertura torácica, el corazón está al alcance de la mano. Por su situación en la caja torácica, la mejor exposición del corazón se da con un corte a lo largo de todo el margen subtorácico (de izquierda a derecha) y el cuerpo estando en posición supina sobreextendida. La imagen resultante asemeja el retrato de las víctimas representadas en la iconografía de Piedras Negras y en el Códice Dresden (figura 3a y b).

A diferencia del acceso por el cual abogamos en este estudio, la esternotoracotomía transversal a nivel del espacio intercostal entre la quinta y sexta costilla,
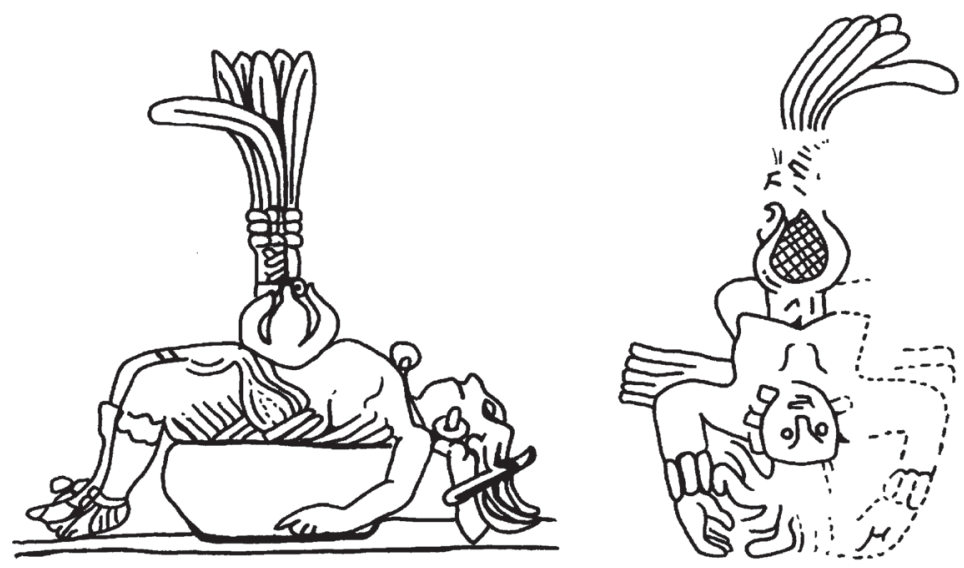

Figura 3. Detalles de Representaciones del Ritual DE EXTRACCIÓN DE CORAZÓN desde una perspectiva lateral: a) estela 11 en Piedras Negras, Guatemala (redibujado a partir de Nájera, 1987);

b) Dresden Codex 3 (3) (redibujado a partir de Thompson, 1988). 
propuesta por Robicsek y Hales (1984: 82), sería obstaculizada por el volumen del seno femenino, complicando y alargando el tiempo de la intervención. Cabe recalcar en este punto que la extracción de corazón no debe entenderse como una intervención quirúrgica (terminología que emplean Robicsek y Hales, 1984: 82) llevada a cabo con el cuidado y con el tiempo necesario, sino el acto culminante de un sacrificio violento que requería una rápida ejecución de una víctima viva y al principio aún consciente.

En lo que se refiere al paso consecutivo, i. e. la remoción cardiaca, se requiere seccionar para ello las membranas que fijan la base del órgano con el tejido conectivo atrás y sobre la columna. Este anclaje se da a la altura de las últimas cuatro vértebras torácicas (T9-T12). Para nuestros propósitos de reconstrucción interesa que esta posición corresponda al patrón que encontramos en las vértebras, lo cual nos permite suponer que las huellas fueron originadas por el impacto violento para liberar el corazón de las membranas y vasos circundantes. A pesar de que no podamos excluir a priori el empleo de otros métodos, la presente reconstrucción conductual sí explica la presencia de las lesiones encontradas en las vértebras torácicas bajas que de otro modo serían difíciles de justificar.

Desde una perspectiva instrumental, el uso de cuchillos filosos es necesario durante la abertura subtorácica para permitir un corte de precisión. Sin embargo, estos no dejarían las marcas de impacto aquí encontradas. Entretanto, hachas o adznes no serían de tanta utilidad para una apertura del tórax (para una discusión sobre los instrumentos, véase Robicsek y Hales, 1984: 69-76; Sievert, 1992: 70-71; Pereira, 1999: 192-194; Talavera, et al., 2001: 32-34). Asumiendo que el implemento cortante no se haya cambiado durante el proceso, tal como nos hacen creer las fuentes y tomando en consideración el tiempo corto de ejecución, podemos pensar que las huellas de impacto fueron producidas por un instrumento que combinaba efectos de corte y de impacto, tales como los cuchillos curvados o las hachas filosas de pedernal, cuya presencia está ampliamente registrada en las escenas de sacrificio.

Al revisar la iconografía del Clásico, nos percatamos en primer lugar de que las representaciones generalmente carecen de precisión anatómica. Por otra parte, las escenas sacrificiales del primer milenio d. C. sí subrayan dos aspectos muy importantes del sacrificio de corazón: la posición sobreextendida de la víctima y la característica herida en el cuerpo. La víctima suele retratarse extendida sobre un altar con el pecho en alto y mantenida en ese lugar mediante sus cuatro extremidades. La porción superior del abdomen (y no el tórax) suele ostentar una profunda apertura que parece convexa en K928 (Robicsek y Hales, 1984: 56) y es reproducida como una sección transversal en los grafitis de Tikal, las pinturas de K1377 y la estela 11 de Piedras Negras (figura 3a). Un detalle relevante puede apreciarse en la estela 14 de Piedras Negras, la cual representa la herida desde una vista diferente (figura 4). Muestra la sección como una amplia apertura angular que converge mesialmente y corre paralela al margen inferior de la caja torácica. Puesta en relación con el procedimiento arriba propuesto, esta 
representación refuerza la hipótesis de que el ritual fue realizado por medio de un corte recto a lo largo de la caja torácica, y no a través de un tajo que haya atravesado la misma.

Tomando en consideración las diferentes líneas de evidencia, las etapas consecutivas del sacrificio de corazón confirman su realización mediante una serie de actos coordinados. En nuestra reconstrucción parece factible que fuera llevado al cabo por una sola persona utilizando un solo implemento, semejante a la descripción de una escena del periodo Colonial en Yucatán (Scholes y Adams, 1938: 106). Al mismo tiempo, la operación resalta el conocimiento anatómico práctico y la destreza del practicante, seguramente un experto que obraba en estrecha coordinación con sus asistentes.

Una importante fuente de información es proporcionada también por la documentación etnohistórica. Las crónicas afirman que una vez extraído el órgano vital, el cuerpo con frecuencia seguía siendo objeto de manipulaciones. Los tratamientos en las ceremonias subsecuentes variaban. Las fuentes señalan el desollamiento, descarnamiento, la amputación de partes del cuerpo (Scholes y Adams, 1938; Edmonson, 1984: 97-98) y la cremación del cuerpo o del corazón como posibles conductas postsacrificiales (Taube, 1994: 650-685). Las manipulaciones póstumas encuentran sustento también en las evidencias óseas de los restos de la acompañante de la Reina Roja en Palenque. La lesiones que afectan varios segmentos vertebrales y de costilla proporcionan, junto con el tronco desalineado, evidencias adicionales para suponer que el cuerpo de la mujer pudo haber sido desmembrado después del ritual sacrificatorio, aspectos todos que demandan una reflexión detenida sobre su presencia y distribución regional en la sociedad maya del Clásico.

Discusión aparte merecen las interrogantes sobre la notoria ausencia de vestigios materiales de procesos sacrificiales en el registro óseo de la región. Hasta hoy, esta falta de evidencia ha obligado a la arqueología a recurrir casi exclusivamente a la evidencia contextual y taxonómica (Coe, 1965: 462-469; Fowler, 1984: 603-618; Welsh, 1988: 167-185; Becker, 1993: 45-50). También el perfil de sexo y edad en el momento de morir ha servido para establecer patrones de muerte no natural, mismo que en algunos casos, como los caches (escondrijos) o los depósitos problemáticos en Becán y Kohunlich, es particularmente notable (Tiesler y Cucina, 2003: 345). Más al norte, la colección del Cenote Sagrado de Chichén Itzá recuperada por Piña Chan presenta una mayoría de individuos masculinos (más del 70 por ciento) cuyo rango de edad se halla entre los 7 y los 15 años (Hooton, 1940: 272-273; Tiesler, 2005a, b: 341-363; Anda, et al., 2004: 380).

Recientemente, la falta de evidencias directas de homicidio ritual ha inducido a una serie de autores a considerar que estos conjuntos mortuorios podrían ser el producto de tratamientos funerarios más que de muertes rituales (Becker, 1992: 185-196, 1993; Chase y Chase, 2003: 256; McAnany, 1995, 60-63; Weiss-Krejci, 2001: 769-780, 2003: 376; Fitzsimmons y Fash, 2003: 300-301). Especialmente Weiss-Krecji (2003: 376) ha expresado sus dudas respecto al origen sacrificial que 
otros investigadores han propuesto para una serie de entierros múltiples mayas no perturbados. Desde la perspectiva de las evidencias esperadas, los resultados encontrados en este estudio son reveladores. El hecho de que las prácticas sacrificiales no dejan obligatoriamente marcas en el esqueleto impone limitaciones al acercamiento tafonómico para su evaluación y limita esta a los hallazgos, como es el caso de este estudio. Tampoco la estimación del estado de articulación en los contextos primarios o el inventario de las partes presentes resulta útil, pues la intervención no da resultado en el disturbio de las partes óseas. Siendo así, la mera ausencia de evidencia de extracción cardiaca no confirma ni rechaza su existencia.

No obstante, la presente reconstrucción sí ayuda a aclarar el por qué las evidencias de violencia perimortem pueden faltar en el registro material. Mientras que el método propuesto por Robicsek y Hales (1984: 82) implica la sección del esternón y probablemente también de las costillas, estas marcas no han sido reportadas hasta el momento en contextos primarios. En contraste, el acercamiento subtorácico transdiafragmático no involucra ningún segmento óseo; así, las huellas vertebrales aquí encontradas son el producto inadvertido de la escisión. Consecuentemente, ausencia de evidencia no significa evidencia de ausencia. Este aspecto, junto con el reducido estado de preservación del material y la escasa atención que las marcas culturales han recibido en la investigación maya, explica la pobreza de información documentada a partir del esqueleto.

Solamente podemos especular sobre la existencia y la forma de muerte violenta a partir de entierros primarios similares a los analizados en este estudio, tal como los numerosos contextos de acompañantes de altos dignatarios mayas durante el Clásico. Por lo pronto, el presente trabajo hace pensar que el sacrifico de los acompañantes fue mucho más común de lo que el registro material ha podido documentar. A la luz de nuestra interpretación no creemos entonces que los actos sacrificiales hayan sido tan aislados entre los mayas del Clásico como algunos autores han señalado.

\section{Conclusión}

Con base en el modelo osteotafonómico de actos rituales, la evidencia contextual y esquelética, concluimos que los practicantes mayas del Clásico eligieron el acceso transdiafragmático subtorácico, abriendo el cuerpo inmediatamente por debajo de la caja torácica. De ser así, las recurrentes marcas de impacto encontradas en los restos de Calakmul, Becán y Palenque constituyen la primera evidencia directa del proceso de sacrificio y manipulación póstuma del cuerpo documentada hasta el momento para la región. El modelo propuesto en este estudio a su vez obligará a revisar la literatura osteológica regional y su registro osteotafonómico, siendo extensivas sus implicaciones a otras áreas y periodos culturales. Al mismo tiempo, la presente reconstrucción explica por qué este ri- 
tual apenas está representado en el registro esquelético. Esta falta de evidencias contundentes subraya a su vez la necesidad de considerar evidencias alternas para distinguir contextos humanos postsacrificiales de aquellos propiamente funerarios. Concluimos por ello que sólo la integración de la evidencia biocultural en un amplio esquema de reconstrucción interdisciplinario ofrecerá una contribución verdadera a la descripción precisa y comprensión de la expresión ritual de los antiguos mayas, como en el caso de los sacrificios humanos.

\section{BIBLIOGRAFÍA}

Anda, Guillermo, Vera Tiesler y Pilar Zavala

2004 "Cenotes, espacios sagrados y la práctica del sacrificio humano en Yucatán", Memorias del XIII Encuentro Internacional de Investigadores de la Cultura Maya, pp. 376-386. Campeche: Universidad Autónoma de Campeche.

Becker, Marshall J.

1992 "Burials as Caches; Caches as Burials: A New Interpretation of the Meaning of Ritual Deposits Among the Classic Period Lowland Maya”, New Theories on the Ancient Maya, pp. 185-196, E. C. Danien y R. J. Sharer (eds.). Filadelfia: University of Pennsylvania (The University Museum Monograph, 77).

1993 "Earth Offering Among the Classic Period Lowland Maya: Burials and Caches as Ritual Deposits", Perspectivas antropológicas en el mundo maya, pp. 45-74, M. J. Iglesias Ponce de León y F. Ligorred Perramón (eds.). Madrid: Sociedad Española de Estudios Mayas.

Brooks, Sheilagh T. y Judy M. Suchey

1990 "Skeletal Age Determination Based on the Os Pubis: A Comparison of the Acsadi-Nemeskeri and Suchey-Brooks Methods", Human Evolution (5): 227238. Holanda: Springer.

Buikstra, Jane E. y Douglas Ubelaker (eds.)

1994 Standards for Data Collection from Human Skeletal Remains. Fayetteville: Arkansas Archaeological Survey (Research Series, 44).

Buikstra, Jane E., T. Douglas Price, Lori E. Wright y James A. Burton

2004 "Tombs from the Copán Acropolis: A Life History Approach", Understanding

Early Classic Copan, pp. 191-212, E. E. Bell, M. A. Canuto y R. Sharer (eds.). Filadelfia: Museum of Archaeology and Anthropology, University of Pennsylvania.

Carrasco, David

1999 City of Sacrifice. The Aztec Empire and the Role of Violence in Civilization. Boston: Beacon Press. 
Carrasco Vargas, Ramón

2000 "El cuchcabal de la Cabeza de la Serpiente", Arqueología Mexicana, VII (42): 12-21. México: Raíces, INAH.

—_ Sylviane Boucher, Paula Álvarez González, Vera Tiesler Blos, Valera García Vierna, Renata García Moreno y Javier Vázquez Negrete

1999 "A Dynastic Tomb from Campeche, Mexico: New Evidence on Jaguar Paw, a Ruler of Calakmul”, Latin American Antiquity, 10 (1): 47-59. Washington, D.C.: Society for American Archaeology.

Chase, Diane, y Arlen Chase

1996 "Maya Multiples: Individuals, Entries and Tombs in Structure A34 of Caracol, Belize”, Latin American Antiquity, 7 (1): 61-79. Washington, D.C.: Society for American Archaeology.

1998 "The Archaeological Context of Caches, Burials, and other Ritual Activities for the Classic Period Maya (as Reflected at Caracol, Belize)", Function and Meaning in Classic Maya Architecture, pp. 299-332, S. Houston (ed.). Washington, D.C.: Dumbarton Oaks.

2003 “Secular, sagrado y 'revisitado’: la profanación, alteración y reconsagración de los antiguos entierros mayas”, Antropología de la eternidad: la muerte en la cultura maya, pp. 255-277, A. Ciudad Ruiz, M. H. Ruz Sosa y M. J. Ponce de León (eds.). Madrid: Sociedad Española de Estudios Mayas, UNAM, Centro de Estudios Mayas.

Coe, William

1965 "Caches and Offertory Practices in the Maya Lowlands", Handbook of Middle American Indians, Vol. 2: The Archaeology of Southern Mesoamerica, pp. 462469, R. Wauchope y G. R. Willey (eds.). Austin: University of Texas.

Cucina, Andrea, Vera Tiesler, y Arturo Romano

2004 "Los acompañantes de Janaab' Pakal y de la 'Reina Roja' de Palenque, Chiapas. El significado de sacrificios humanos de exequias en la sociedad maya del Clásico", Janaab’ Pakal de Palenque. Vida y muerte de un gobernante maya, pp. 71-102, V. Tiesler y A. Cucina (eds.). México: UNAM, UADY.

Duday, Henry

1997 “Antropología biológica de 'campo’, tafonomía y arqueología de la muerte”, El cuerpo humano y su tratamiento mortuorio, pp. 91-126, E. Malvido, G. Pereira y V. Tiesler (eds.). México: INAH (Colección Científica).

Duday, Henry, Patrice Courtaud, Eric Crubezy, Pascal Sellier y Anne-Marie Tillier 1990 "L'anthropologie 'de terrain': reconnaissance et interprétation des gestes funéraires”, Bulletins et Mémoires de la Société d'Anthropologie de Paris, 2 (3-4): 29-50.

Edmonson, Munro S.

1984 "Sacrifice in the Books of Chilam Balam of Tizimin and Chumayel", Ritual 
Human Sacrifice in Mesoamerica, pp. 91-100, E. H. Boone (ed.). Washington, D.C.: Dumbarton Oaks.

Fitzsimmons, James L. y William L. Fash

2003 “Susaj B’aak: muerte y ceremonia mortuoria en la Plaza Mayor de Copán”, Antropología de la eternidad: la muerte en la cultura maya, pp. 299-315, A. Ciudad Ruiz, M. H. Ruz Sosa y M. J. Ponce de León (eds.). Madrid: Sociedad Española de Estudios Mayas, UNAM, Centro de Estudios Mayas.

Fowler, William R.

1984 "Late Preclassic Mortuary Patterns and Evidence for Human Sacrifice at Chalchuapa, El Salvador”, American Antiquity, 49: 603-618. Washington, D.C.: Society for American Archaeology.

García Moreno, Renata, y Josefina Granados

2000 "Tumbas reales de Calakmul”, Arqueología Mexicana, VII (42): 28-33. México: Raíces, INAH.

Gillespie, Susan

2001 "Personhood, Agency, and Mortuary Ritual: A Case Study from the Ancient Maya”, Journal of Anthropological Archaeology, 20 (1): 73-112. Nueva York: Academic Press.

2002 "Body and Soul Among the Maya: Keeping the Spirits in Place", The Space and Place of Death, pp. 67-78, H. Silverman y David Small (eds.). Arlington: American Anthropological Association (Archaeological Papers of the American Anthropological Association, 10).

Gómez, María José, Abel Morales López, Vera Tiesler y William Folan

2003 "Ritual y mutilación póstuma del cuerpo humano durante el posclásico: nuevas evidencias de Champotón, Campeche”, Mexicon, 25: 146-147. Markt Schwaben: Verlag Anton Saurwein.

González Cruz, Arnoldo

1998 "El Templo de la Reina Roja, Palenque, Chiapas”, Arqueología Mexicana, V (30): 61. México: Raíces, INAH

2001 "The Red Queen”, Pre-Columbian Art Research Institute, San Francisco. (www.mesoweb.com/palenque/features/red_queen/01.html).

González Torres, Yolotl

1985 El sacrificio humano entre los mexicas. México: INAH, FCE.

2003 "El sacrificio humano entre los mexicas", Arqueología Mexicana, XI (63): 4045. México: Raíces, INAH.

Hooton, Earnest A.

1940 "Skeletons from the Cenote of Sacrifice at Chichen Itza", The Maya and 
Their Neighbors: Essays on Middle American Anthropology and Archaeology, pp. 272-280, C. L. Hay, R. L. Linton, S. K. Lothrop, H. L. Shapiro y G. C. Vaillant (eds.). Nueva York: Appleton-Century.

Houston, Stephen, Héctor L. Escobedo, Andrew Scherer, Mark Child

y James L. Fitzsimmons

2003 "Classic Maya Death at Piedras Negras, Guatemala”, Antropología de la eternidad: la muerte en la cultura maya, pp. 113-143, A. Ciudad Ruiz, M. H. Ruz Sosa, y M. J. Ponce de León (eds.). Madrid: Sociedad Española de Estudios Mayas, UNAM, Centro de Estudios Mayas.

Iscan, Mehmet Y. y Susan R. Loth

1986 "Estimation of Age and Determination of Sex from the Sternal Rib", Forensic Osteology: Advances in the Identification of Human Remains, pp. 68-89, K. J. Reichs (ed.). Springfield, Illinois: C. C. Thomas.

Kampen, Michael

1978 "The Graffiti of Tikal, Guatemala", Estudios de Cultura Maya, IX: 155-174. México: UNAM, IIFL, Centro de Estudios Mayas.

Landa, Diego de, fray

1982 Relación de las cosas de Yucatán [1566]. México: Porrúa.

López Austin, Alfredo

1989 Cuerpo humano e ideología. Las concepciones de los antiguos nahuas. México: UNAM.

1998 “Los ritos. Un juego de definiciones”, Arqueología Mexicana, VI (34): 4-17. México: Raíces, INAH.

López Jiménez, Fanny y Arnoldo González Cruz

1995 "El Templo de la Reina Roja en Palenque, Chiapas", CIHMECH, 5: 121-134. México: UNAM, Coordinación de Humanidades.

Lothrop, Samuel K.

1952 Metals from the Cenote of Sacrifice, Chichen Itza, Yucatan. Cambridge, Massachusetts: Peabody Museum of American Archaeology and Ethnology (Memoirs, vol. 10, núm. 2).

Martin, Simon y Nikolai Grube

2000 Chronicle of the Maya Kings and Queens. Londres: Thames and Hudson.

Massey, Virginia K. y Gentry Steele

1982 "Preliminary Notes on the Dentition and Taphonomy of the Colha Human Skeletal Material”, Archaeology at Colha, Belize: The 1981 Interim Report, pp. 198202, T. R. Hester (ed.). San Antonio: University of Texas.

1997 "A Maya Skull Pit from the Terminal Classic Period, Colha, Belize", Bones of the Maya: Studies of Ancient Skeletons, pp. 62-77, S. L. Whittington y D. M. Reed (eds.). Washington, D.C.: Smithsonian Institution. 
Matos Moctezuma, Eduardo

1986 Muerte a filo de obsidiana. México: SEP (Lecturas Mexicanas, 50).

McAnany, Patricia A.

1995 Living with the Ancestors. Kinship and Kingship in Ancient Maya Society. Austin: University of Texas.

1998 "Ancestors and the Classic Maya Built Environment", Function and Meaning in Classic Maya Architecture, pp. 271-298, S. Houston (ed.). Washington, D.C.: Dumbarton Oaks.

y Sandra López Varela

1999 "Re-creating the Formative Maya Village of K'axob: Chronology, Ceramic Complexes, and Ancestors in Architectural Context", Ancient Mesoamerica, 10 (1): 147-168. Cambridge, Inglaterra: Cambridge University Press.

—

1999 "Mortuary Ritual and Family Politics at Formative and Early Classic K'axob, Belize”, Ancient Mesoamerica, 10 (1): 129-146. Cambridge, Inglaterra: Cambridge University Press.

Meindl, Richard S., C. Owen Lovejoy, Robert P. Mensforth y Robert A. Walker

1985 "A Revised Method of Age Determination Using the Os Pubis, with a Review and Tests of Accuracy of Other Current Methods of Pubic Symphysial Aging”, American Journal of Physical Anthropology, 68 (1): 29-45. Columbus: American Association of Physical Anthropologists.

Miller, Virginia E.

2003 "Representaciones de sacrificio en Chichén Itzá”, Antropología de la eternidad: la muerte en la cultura maya, pp. 383-404, A. Ciudad Ruiz, M. H. Ruz Sosa y M. J. Ponce de León (eds.). Madrid: Sociedad Española de Estudios Mayas, UNAM, Centro de Estudios Mayas.

y Karl Taube

1993 The Gods and the Symbols of Ancient Mexico and the Maya. Londres: Thames and Hudson.

Nájera, Martha Ilia

1987 El don de la sangre en el equilibrio cósmico. México: UNAM, Centro de Estudios Mayas.

Pereira, Grégory

1999 Potrero de Guadalupe: anthropologie funéraire d'une communauté pré-tarasque du nord du Michoacán, Mexique. Oxford: British Archaeological Reports (International Series, 816).

Pijoan Aguadé, Carmen

1997 "Evidencias de sacrificio humano y canibalismo en restos óseos: el caso del 
entierro número 14 de Tlatelolco, D.F.”. Tesis de doctorado en Antropología, México: UNAM.

Posel, Peter (ed.)

1991 Sobotta. Spielend durch die Anatomie. Munich: Urban \& Schwarzenberg.

Robicsek, Francis y Donald M. Hales

1984 "Maya Heart Sacrifice: Cultural Perspective and Surgical Technique", Ritual Human Sacrifice in Mesoamerica, pp. 49-90, E. H. Boone (ed.). Washington, D.C.: Dumbarton Oaks.

Ruz, Alberto

1973 El Templo de las Inscripciones, Palenque. México: INAH (Colección Científica).

1991 Costumbres funerarias de los antiguos mayas. México: UNAM, IIFL, Centro de Estudios Mayas.

Schele, Linda

1984 "Human Sacrifice Among the Classic Maya", Ritual Human Sacrifice in Mesoamerica, pp. 7-48, E. H. Boone (ed.). Washington, D.C.: Dumbarton Oaks.

y Mary Ellen Miller

1986 The Blood of Kings. Nueva York: G. Baziller.

Scholes, France V. y Eleanor Adams

1938 Don Diego Quijada Alcalde Mayor de Yucatán (1561-1565). México: José Porrúa. Vol. 1.

Sievert, April K.

1992 Maya Ceremonial Specialization. Lithic Tools from the Sacred Cenote at Chichén Itzá, Yucatán. Madison: Prehistory Press (Monographs in World Archaeology, 12).

Stuart, David

2003 “La ideología del sacrificio entre los mayas”, Arqueología Mexicana, XI (63): 24-29. México: Raíces, INAH.

Talavera, Jorge, Juan Martín Rojas y Enrique García

2001 Modificaciones culturales en los restos óseos de Cantona, Puebla. Un análisis bioarqueológico. México: INAH (Colección Científica).

Taube, Karl

1994 "The Birth Vase: Natal Imagery in Ancient Maya Myth y Ritual", The Maya Vase Book, vol. 4, pp. 650-685, Justin Kerr (ed.). Nueva York: Kerr Associates.

1998 "The Iconography of Toltec Period Chichen Itza”, Hidden Among Hills. Maya Archaeology of the Northwestern Yucatan Peninsula, pp. 212-246, H. J. Prem (ed.). Möckmühl: Verlag Von Flemming (Acta Mesoamericana, 7). 
Thompson, Eric S.

1988 Un comentario al Códice de Dresde. Libro de jeroglifos mayas, trad. de Jorge Ferreiro Santana. México: FCE.

Tiesler, Vera

2002a “Un caso de decapitación prehispánica de Calakmul, Campeche”, Antropología Física Latinoamericana, 3: 129-142. México: Asociación Latinoamericana de Antropología Biológica, UNAM, Instituto de Investigaciones Antropológicas.

2002b Análisis de los restos óseos y dentales humanos recuperados en el sitio arqueológico de Becán, Campeche, durante las temporadas 1999-2001, reporte inédito. México: INAH.

2003a Análisis del material óseo humano procedente de Calakmul, Campeche, reporte inédito. Campeche: INAH.

2005a "Mortuary Treatments in Classic Maya Elite Burials. An Osteo-Taphonomic Perspective", Continuity and Change. Maya Religious Practices in Temporal Perspective, pp. 143-156, D. Graña Behrens, N. Grube, C. M. Prager, F. Sachse, S. Teufel y E. Wagner (eds.). Markt Schwaben: Anton Saurwein.

2005b "What Can the Bones Really Tell Us? The Study of Human Skeletal Remains from Cenotes", Stone Houses and Earth Lords. Maya Religion in the Cave Context, pp. 341-363, Keith M. Prufer y James E. Brady (eds.). Boulder: University of Colorado.

y Andrea Cucina

2003 "Sacrificio, tratamiento y ofrenda del cuerpo humano entre los mayas del Clásico: una mirada bioarqueológica”, Antropología de la eternidad: la muerte en la cultura maya, pp. 337-354, A. Ciudad Ruiz, M. H. Ruz Sosa y M. J. Ponce de León (eds.). Madrid: Sociedad Española de Estudios Mayas, UNAM, Centro de Estudios Mayas.

\footnotetext{
_ y Luz Evelia Campaña

2006 "Sacrificio y tratamiento ritual del cuerpo humano en la antigua sociedad maya: el caso del Depósito E-1003 de Becán, Campeche”, Arqueología, 33: 32-46. México: INAH.

- Andrea Cucina y Arturo Romano

2002 "Vida y muerte del personaje hallado en el Templo XIII-sub, Palenque: I. Culto funerario y sacrificio humano", Mexicon, XXIV (4): 75-78. Markt Schwaben: Verlag Anton Saurwein.

Andrea Cucina y Arturo Romano

2004 "Who was the Red Queen? Identity of the Female Maya Dignitary from the Sarcophagus Tomb of Temple XIII, Palenque, Mexico", HOMO. Journal of Comparative Human Biology, 55 (1-2): 65-76. Jena: Australasian Society for Human Biology.
} 
Tozzer, Alfred M.

1941 Landa's Relación de las cosas en Yucatán: a translation. Cambridge, Massachusetts: Harvard University (Papers of the Peabody Museum of American Archaeology and Ethnology, 18).

Ubelaker, Douglas H.

1989 "The Estimation of Age at Death from Immature Human Bones", Age Markers in the Human Skeleton, pp. 55-70, M. Y. Iscan (ed.). Springfield, Illinois: C. C. Thomas.

Weiss-Krejci, Estella

2001 "Restless Corpses: 'Secondary Burial' in the Babenberg and Habsburg Dynasties”, Antiquity, 75 (290): 769-780. York: Antiquity Trust.

2003 "Victims of Human Sacrifice in MultipleTombs of the Ancient Maya: a Critical Review", Antropología de la eternidad: la muerte en la cultura maya, pp. 355-381, A. Ciudad Ruiz, M. H. Ruz Sosa y M .J. Ponce de León (eds.). Madrid: Sociedad Española de Estudios Mayas, UNAM, Centro de Estudios Mayas.

Welsh, Bruce

1988 An Analysis of Classic Lowland Maya Burials. Londres: British Archaeological Reports (International Series, 409). 